\title{
Gallenuntersuchungen nach Phosphor- und Arsenvergiftung. Von
}

Dr. philos. Alfons Pilzecker.

Der Redaktion zugegangen am 25. Januar 1904.

\section{$\S 1$.}

Nachstehende Untersuchung setzt sich das Ziel, zu den von Prof. Brauer so erfolgreich in Angriff genommenen experimentell pathologischen Studien der Veränderungen, welche die Galle unter der Einwirkung krankmachender Stoffe erfährt, einen Beitrag zu liefern. Und zwar hat Verfasser den Einfluß untersucht, den eine chronische Vergiftung durch Phosphor und durch Arsen auf die Gallenbeschaffenheit hat.

Die außerordentlichen individuellen Schwankungen, welche gerade die Gallensekretion zeigt, machen es, wie Stadelmann schon bemerkt, nötig, "stets wieder von neuem, sobald man Versuche an Gallenfistelhunden machen will, die normale Ausscheidungsgröße für das betreffende Tier genau festzustellen». Verfasser hat daher, bevor er mit der Intoxikation seiner Versuchstiere begann, Versuchsreihen vorausgeschickt, in denen die dem gesunden Tier entnommene Galle den gleichen Prüfungen unterworfen wurde, die später mit der von dem vergifteten Tiere sezernierten Galle angestellt werden sollten.

Bei der Anknüpfung dieser Untersuchungen an die Brauersche Arbeit war es verständlich, daß zunächst auf das pathologische Auftreten von Eiweiß und Zucker in der Galle gefahndet wurde.

Zum Nachweis von koagulierbarem Eiweiß in der Galle steht zunächst das von Brauer ausgearbeitete und Bd. XL, S. 203 dieser Zeitschrift beschriebene Verfahren zur Verfügung. Es gelingt hiermit, das in der Hundegalle enthaltene echte Mucin in Lösung zn erhalten, während anderes etwa auftretendes koagulierbares Eiweiß gefällt wird. 
Da Brauer mit Hilfe dieser Methode gezeigt hat, daß koagulierbares Eiweiß in der dem lebenden, gesunden Tier entnommenen Galle nicht vorkommt, so wird man sein Auftreten als die Folge pathologischer Verhältnisse der Gallensekretion zu deuten berechtigt sein.

Eine weitere Methode, sich über das Auftreten von vermehrtem Eiweiß in der Galle Aufschluß zu verschaffen, bietet die Stickstoffbestimmung des Alkoholniederschlages der Galle nach Kjeldahl.

Da aber dieser Alkoholniederschlag neben etwaigem anderweitigen Eiweiß stets auch das Mucin der Galle enthält, da ferner die Gallenfarbstoffe in wechselnder Menge darin enthalten sind, so sind diese Eiweißstickstoffwerte nicht eindeutig. Durch konstantes Einführen der Kanüle bis zu möglichst großer Tiefe kann man darnach streben, den Mucinanteil, der größtenteils den letzten abführenden Gallenwegen entstammt, möglichst einzuschränken, während man zur Prüfung der Konstanz in der Farbstoffbeimischung auf das Auge angewiesen ist.

Ebenfalls nach der Kjeldahl-Methode haben wir ferner an jeder Gallenprobe den Gesamtstickstoffgehalt festgestellt. Neben den schon in der vorigen Probe auf Eiweißstickstoff enthaltenen Körpern kommen hier als normal schon in der Galle enthaltene Bestandteile in Betracht: Gallenfarbstoffe, Lecithin, Gallensäuren und Harnstoff. $\mathrm{Ob}$ pathologisch noch andere $\mathrm{N}$-haltige Körper in der Galle auftreten können, etwa wie Ammoniak im Harn, darüber ist wenig bekannt. Nur Lecithin und Tyrosin sollen bei akuter gelber Leberatrophie und bei Typhus beobachtet worden sein.

Weit einfacher als der Nachweis von Eiweiß in der Galle ist derjenige von Zucker, dessen Vorkommen in der normalen Galle von einigen Forschern wie Naunyn und Harley behauptet, durch Brauers Versuch jedoch widerlegt worden ist. Wir bedienten uns der auch von Brauer angewandten Fehlingschen Probe.

Um ein objektives Maß für den Grad der Dickflüssigkeit der Galle zu haben, wurde das Viskosimeter zu Hilfe gezogen. Es war dies um so wünschenswerter, als viskosimetrische Be- 
stimmungen der Galle bisher nicht vorliegen. Verfasser bediente sich hierzu des von André Mayer im Jahrgang 1901 der Société de Biologie S. 1140 beschriebenen sehr einfachen Apparates. Die Kapazität der Aufnahmekugel betrug einschießlich der U-förmigen Glasröhre für unser Viskosimeter 2,2 ccm.

Der vorher festgestellte Viskositätswert für destilliertes Wasser ergab für dasselbe, gemessen in einem Wasserbade von $40^{\circ} \mathrm{C}$., genau 20,0 sec.; auf diesen sind also die später mitgeteilten Werte der Gallenviskosität zu beziehen.

Das spezifische Gewicht wurde mit der Mohrschen Wage ermittelt. Schließlich wurde, im Hinblick auf die bemerkenswerten Befunde, die Prof. Brauer bei seinen Versuchen mit Alkoholintoxikation erhoben hat, stets ein Quantum Galle nach gehöriger Verdünnung mit physiologischer Kochsalzlösung zentrifugiert und das Sediment mikroskopisch untersucht.

\section{$\S 2$. Versuchsanordnung.}

Als Versuchstiere wurden zwei größere Hunde von 18 resp. $20 \mathrm{~kg}$ Gewicht gewählt und beiden Tieren am gleichen Tage, dem 22. V. 03, Gallenfisteln mit Vernähung der vorgelagerten Gallenblasenwand an die Bauchwand angelegt. Die Einmündung des Choledochus in das Duodenum wurde geschont, sodaß der Abfluß von Galle in den Darm gewahrt blieb. Da die Tiere sich außerdem die abfließende Galle gegenseitig gierig ableckten, so steht zu erwarten, daß das *Gallensäuregleichgewicht», um mit Brauer zu reden, bei unseren Versuchen genügend gewahrt ist.

Nach sicherer Vernarbung wurden am 16. VI., also 25 Tage nach der Operation, mit dem Hunde tastende Versuche begonnen. Bei der Hündin erlitten dieselben eine unliebsame Unterbrechung, da blutendes Narbengewebe eine kleine Nachoperation nötig machte. Nach Beseitigung desselben und abgelaufener Heilung konnten am 13. VII. die Versuche auch mit diesem Tier fortgeführt werden.

Zur Durchführung aller im vorigen Paragraphen angegebenen Bestimmungen waren jedesmal etwa $20 \mathrm{ccm}$ Galle nötig. Um diese Menge zu gewinnen, wurde das Versuchstier in der auch 
von Prof. Brauer benutzten und beschriebenen Vorrichtung aufgestellt gehalten, bis mindestens obengenanntes Quantum durch die eingeführte Kanüle in den angehängten Glaskolben gelaufen war.

Wie bereits oben angeführt, wurde dabei die Kanüle bis zu einer bestimmten Marke eingeführt und stetig kontrolliert, daß sie sich nicht aus der Fistel herausschob, während sie durch Binden in ihrer Stellung fixiert gehalten war. Und zwar gelang es bei dem Hunde, bis $15 \mathrm{~cm}$ vom Ausgang der Fistel an gerechnet einzugehen, während bei der Hündin dies Maß $12 \mathrm{~cm}$ war. Die abfließende Galle wurde ständig überwacht, sodaß sich jede Beimischung von Blut oder Schleimflocken sofort verraten mußte. Bei dem Phosphorhund hat gegen das Ende der Vergiftung hin durch Katarrh der Gallenwege bedingte Vermehrung der Schleimabsonderung stattgefunden, denn es zeigten sich dicke Massen wenig gefärbten Schleimes am Ausgang der Fistel. Dieser vermehrte Schleim gelangte jedoch nicht in die Kanüle und wurde stets sorgsam entfernt, um seinen Übertritt in den übrigens durch Gummistöpsel verschlossenen Glaskolben zu verhindern.

Die aufgefangene Galle wurde stets sofort in unmittelbarem Anschluß an die Entnahme derselben verarbeitet. Wir dürfen also durchaus sicher sein, daß irgendwelche Umsetzungsprozesse an derselben noch nicht Platz gegriffen haben.

\section{§3. Untersuchung normaler Galle.}

In der folgenden Tabelle I sind die Werte zusammengestellt, welche die Prüfung der dem gesunden Hunde durch die Gallenfistel abgeflossenen Galle ergab.

$\mathrm{Zu}$ den Zahlen der Tabelle ist wenig hinzuzufügen:

Die notierten Gallenmengen zeigen die erwarteten, großen Schwankungen. Die besonders hohen Zahlen der letzten beiden Beobachtungstage, an denen die Gallenentnahme nicht morgens, sondern nachmittags stattfand, erklärt sich wohl im Sinne der Pawlowschen Untersuchungen aus der eingetretenen Fettverdauung, die dem vormittags nüchternen Hunde fehlte. 
Gallenuntersuchungen nach Phosphor- und Arsenvergiftung. 161

Tabelle I.

\begin{tabular}{|c|c|c|c|c|c|c|}
\hline Tageszeit & $\begin{array}{l}\text { 23. Juni } \\
\text { morgens } \\
8^{1} / 2-12 \mathrm{Uhr}\end{array}$ & $\begin{array}{c}\text { 25. Juni } \\
\text { morgens } \\
8^{1 / 2} / 2-10^{1 / 3} \text { Uhr }\end{array}$ & $\begin{array}{l}\text { 1. Juli } \\
\text { morgens } \\
9-11 \text { Uhr }\end{array}$ & $\begin{array}{l}\text { 4. Juli } \\
\text { morgens } \\
9-11 \text { Uhr }\end{array}$ & $\begin{array}{l}\text { 6. Juli } \\
\text { nachm. } \\
\text { 3-4 Uhr }\end{array}$ & $\begin{array}{l}\text { 7. Juli } \\
\text { nachm. } \\
4^{1} / 2-5 \mathrm{Uhr}\end{array}$ \\
\hline $\begin{array}{l}\text { Gewonnene } \\
\text { Gallenmenge }\end{array}$ & $57 \mathrm{ccm}$ & $43 \mathrm{ccm}$ & $40 \mathrm{ccm}$ & $28 \mathrm{ccm}$ & $50 \mathrm{ccm}$ & $28 \mathrm{ccm}$ \\
\hline Farbe & $\begin{array}{c}\text { Grünlich-braun } \\
\text { klar }\end{array}$ & $\begin{array}{l}\text { Unver- } \\
\text { ändert }\end{array}$ & $\begin{array}{c}\text { Bräunlich-grün, } \\
\text { dunkler }\end{array}$ & $\begin{array}{c}\text { Nicht } \\
\text { dunkler, } \\
\text { aber grüner } \\
\text { als bisher } \\
\end{array}$ & $\begin{array}{c}\text { Heller, mehr } \\
\text { bräunlich }\end{array}$ & $\begin{array}{c}\text { Relativ hell, } \\
\text { bräunlich }\end{array}$ \\
\hline Konsistenz & Wenig dick & Desgl. & $\begin{array}{l}\text { Etwas dicker } \\
\text { als bisher }\end{array}$ & Dicker & $\begin{array}{c}\text { Kaum } \\
\text { dünner als } \\
\text { yorgestern } \\
\end{array}$ & $\begin{array}{l}\text { Nur wenig } \\
\text { dünner als } \\
\text { gestern }\end{array}$ \\
\hline $\begin{array}{c}\text { Sichtbare Bei- } \\
\text { mischungen }\end{array}$ & $\begin{array}{c}\text { Weder Blut } \\
\text { noch Schleim }\end{array}$ & $\begin{array}{l}\text { Eein Blut, } \\
\text { noch } \\
\text { Schleim }\end{array}$ & $\begin{array}{c}\text { Kein Blut, } \\
\text { noch Schleim }\end{array}$ & $\begin{array}{c}\text { Kein Blut, } \\
\text { etwas } \\
\text { Schleim }\end{array}$ & $\begin{array}{l}\text { Kein Blut, } \\
\text { etwas } \\
\text { Schleim }\end{array}$ & $\begin{array}{l}\text { Kein Blut, } \\
\text { wenig } \\
\text { Schleim }\end{array}$ \\
\hline Reaktion & Alkalisch & $\begin{array}{c}\text { Alkalisch } \\
\text { : }\end{array}$ & Alkalisch & Alkalisch & Neutral & $\begin{array}{l}\text { Schwach } \\
\text { alkalisch }\end{array}$ \\
\hline $\begin{array}{l}\text { Spezif. } \\
\text { Gewicht }\end{array}$ & 1016 & 1015 & 1018 & 1023 & 1028 & 1024 \\
\hline Viskosität & - & - & 56,1 & 62,2 & 68,1 & 56,4 \\
\hline Kochprobe & Negativ & Negativ & Negativ & \begin{tabular}{|c|} 
Keine \\
Fällung,aber \\
Spur \\
Opaleszenz \\
\end{tabular} & \begin{tabular}{|c|} 
Kein Nieder- \\
schlag, aber \\
schwache \\
Opaleszenz \\
\end{tabular} & $\begin{array}{c}\text { Ganz geringe } \\
\text { Spur } \\
\text { Opaleszenz } \\
\end{array}$ \\
\hline Zuckerprobe & Negativ & Negativ & Negativ & Negativ & Negativ & Negativ \\
\hline $\begin{array}{c}\text { Gesamt-N in } \\
5 \mathrm{ccm}\end{array}$ & - & - & $13,16 \mathrm{mg}$ & $13,16 \mathrm{mg}$ & $14,92 \mathrm{mg}$ & $13,86 \mathrm{mg}$ \\
\hline $\begin{array}{l}\text { Stickstoff des } \\
\text { Alk.-Niederschl. } \\
\text { in } 5 \mathrm{ccm} \\
\end{array}$ & - & - & $4,76 \mathrm{mg}$ & $3,36 \mathrm{mg}$ & $3,08 \mathrm{mg}$ & $2,24 \mathrm{mg}$ \\
\hline $\begin{array}{l}\text { Mikroskopi- } \\
\text { scher Befund }\end{array}$ & $\begin{array}{l}\text { Detritus, Farb- } \\
\text { massen, nichts } \\
\text { Histologisches }\end{array}$ & $\begin{array}{l}\text { Wie } \\
\text { gestern }\end{array}$ & $\mid \begin{array}{c}\text { Nichts Histologi- } \\
\text { sches, nur ge- } \\
\text { färbter Detritus, } \\
\text { einige Kristalle }\end{array}$ & $\begin{array}{c}\text { Keine } \\
\text { Zellen, keine } \\
\text { Zylinder }\end{array}$ & $\begin{array}{c}\text { Derselbe } \\
\text { Befund wie } \\
\text { Irüher }\end{array}$ & $\begin{array}{l}\text { Wie } \\
\text { früher }\end{array}$ \\
\hline
\end{tabular}

Die Tabelle ergibt ferner ein Ansteigen der Wertè des spezifischen Gewichts, sowie die Visknositätswerte. Das höchste spezifische Gewicht 1028 wurde an demselben Nachmittag gefunden, an welchem durch die lebhafte Verdauung des Tieres

Hoppe-Seyler's Zeitschrift f. physiol. Chemic. XLI. 
die Absonderungsgeschwindigkeit und ebenso die Viskosität besonders groß war. Daß während der Verdauung mehr Salze mit der Galle sezerniert werden, dagegen weniger Mucin, Taurocholsäure und Ätherextraktstoffe, hat schon HoppeSeyler angegeben. R. Thomas hat es in seiner Arbeit «Über die Abhängigkeit der Absonderung und Zusammensetzung der Galle von der Nahrung» (Straßburg 1890) für Fleischnahrung bestätigt.

Die Kjeldahl-Bestimmungen ergaben für den Gesamtstickstoff eine gute Konstanz, während der Stickstoff des Alkoholniederschlags innerhalb der 4 untersuchten Tage, die den Zeitraum einer Woche umspannen, bis unter die Hälfte des Anfangwertes allmählich herabgesunken ist. -- Einige Worte noch über die Resultate der qualitativen Proben auf koagulierbares Eiweiß. Während sich an den 3 ersten Tagen beim Kochen der vorsichtig, aber bis zur deutlich sauren Reaktion angesäuerten, klar gebliebenen Galle auch nicht die geringste Veränderung zeigte, ist an den 3 letzten Tagen eine Spur Opaleszenz bemerkt. Dieselbe ist jedoch nicht im entferntesten zu vergleichen mit dem, was sich uns später als Trübung zeigte und ganz deutlich auf gefälltes Eiweiß gedeutet werden mußte. Die Farbenbestimmungen zeigen, da $\beta$ im allgemeinen bei großer Absonderungsgeschwindigkeit die Galle weniger Farbstoff enthält, und daß im übrigen bald der grünfärbende Faktor vor dem rotbraunfärbenden in den Vordergrund tritt, bald umgekehrt. Wir werden das bei den später zu schildernden Versuchen mit pathologischer Galle noch deutlicher bemerken können.

Wir lassen hier zunächst die Werte folgen, welche das zweite Versuchstier, die Hündin, bei den analog ausgeführten Versuchen darbot.

Ein Vergleich mit Tabelle I lehrt, daß für die Hündin alle Werte eine größere Konstanz zeigen als für den Hund. Die Gallenmenge in der Zeiteinheit ist für alle Tage ungefähr die gleiche.

Die Kochproben auf koagulierbares Eiweiß, die vor der Nachoperation wegen Anwesenheit von Blut selbstverständlich Trübung ergeben hatten, fielen nachher absolut negativ aus. 
Tabelle II.

\begin{tabular}{|c|c|c|c|c|c|}
\hline $1^{\text {Tageszeit }}$ & $\begin{array}{c}\text { 2. Juli } \\
\text { vorm. } \\
10^{1} / 2-1 \mathrm{Uhr}\end{array} \mid$ & $\begin{array}{l}\text { 6. Juli } \\
\text { vorm. } \\
9-11 \mathrm{Uhr}\end{array}$ & $\begin{array}{l}\text { 7. Juli } \\
\text { vorm. } \\
\text { 8-11 Uhr }\end{array}$ & $\begin{array}{l}\text { 13. Juli } \\
\text { nachm. } \\
4-6 \mathrm{Uhr}\end{array}$ & $\begin{array}{l}\text { 14. Juli } \\
\text { nachm. } \\
4-5^{1} / 4 \mathrm{Uhr}\end{array}$ \\
\hline $\begin{array}{l}\text { Gewonnene } \\
\text { Gallenmenge }\end{array}$ & $20 \mathrm{ccm}$ & $20 \mathrm{ccm}$ & $\begin{array}{l}\text { Wenige } \\
\text { Tropfen }\end{array}$ & $20 \mathrm{ccm}$ & $25 \mathrm{ccm}$ \\
\hline Farbe & $\begin{array}{c}\text { Grünlich- } \\
\text { braun, klar }\end{array}$ & Bräunlich & $\begin{array}{c}\text { Nicht } \\
\text { dunkler als } \\
\text { gestern }\end{array}$ & $\begin{array}{l}\text { Auffallend hell, } \\
\text { gelblich-braun }\end{array}$ & $\begin{array}{c}\text { Hell, } \\
\text { gelbbraun }\end{array}$ \\
\hline Konsistenz & $\begin{array}{l}\text { Mäßig } \\
\text { dick }\end{array}$ & $\begin{array}{l}\text { Dünn- } \\
\text { flüssig }\end{array}$ & \multirow{3}{*}{\begin{tabular}{|c|} 
Nicht dicker \\
als gestern \\
Dilatation \\
der Fistel \\
Versuche \\
werden \\
wegen Blu- \\
tung aus der \\
Fistel unter- \\
brochen \\
Nach- \\
operation
\end{tabular}} & Dünnflüssig & Dünnflüssig \\
\hline $\begin{array}{c}\text { SichtbareBei- } \\
\text { mischungen }\end{array}$ & $\begin{array}{c}\text { Deutlich } \\
\text { mehrere } \\
\text { Blutkoagula }\end{array}$ & $\begin{array}{l}\text { Deutliche } \\
\text { Blutbei- } \\
\text { mischung }\end{array}$ & & $\begin{array}{c}\text { Frei von Blut } \\
\text { und sichtbarem } \\
\text { Schleim }\end{array}$ & $\begin{array}{c}\text { Frei von Blut } \\
\text { und sichtbarem } \\
\text { Schleim }\end{array}$ \\
\hline Reaktion & Alkalisch & Alkalisch & & Alkalisch & Alkalisch \\
\hline Spezif. Gew. & 1012 & 1016 & - & 1014 & 1015 \\
\hline Viskosität & 55,2 & 46,2 & - & 26,7 & 21,7 \\
\hline Kochprobe & $\begin{array}{l}\text { Leichte } \\
\text { Trübung }\end{array}$ & Trübung & - & Ganz negativ & Ganz negativ \\
\hline Zuckerprobe & Negativ & 一 & - & Negativ & Negativ \\
\hline $\begin{array}{l}\text { Stickstoff des } \\
\text { Alk.-Niederschl. } \\
\text { in } 5 \mathrm{ccm}\end{array}$ & $7,14 \mathrm{mg}$ & - & - & $7,70 \mathrm{mg}$ & $6,86 \mathrm{mg}$ \\
\hline $\begin{array}{c}\text { Gesamt-N in } \\
5 \mathrm{ccm}\end{array}$ & $2,66 \mathrm{mg}$ & - & - & $0,98 \mathrm{mg}$ & $0,84 \mathrm{mg}$ \\
\hline $\begin{array}{l}\text { Mikroskopi- } \\
\text { scher Befund }\end{array}$ & $\begin{array}{c}\text { Keine histo- } \\
\text { logische Ele- } \\
\text { mente in der } \\
\text { blutfreien } \\
\text { Probe }\end{array}$ & - & 一 & $\begin{array}{c}\text { Keine Zellen } \\
\text { noch Zylinder, } \\
\text { nur Detritus, } \\
\text { Farbstoff, Kri- } \\
\text { stalle }\end{array}$ & $\begin{array}{l}\text { Derselbe Befnnd } \\
\text { wie gestern, } \\
\text { keine Zellen, } \\
\text { keine Zylinder }\end{array}$ \\
\hline
\end{tabular}

Ein Vergleich der N-Werte aus der Galle des Hundes mit denen der Hündin zeigt die weiten, für die Gallensekretion bestehenden individuellen Unterschiede. Dieselben betreffen auch die Viskosität; da die beiden zuerst gefundenen Werte 
wegen Blutbeimischung nicht in Betracht kommen, so muß man die Zahlen 26 und 27 der beiden letzten Versuchstage, also die normalen, für die Hündin ansehen. Während also für dieses Tier die Viskosität der Galle wenig höher war als die des destillierten Wassers, ist sie bei dem Hunde durchschnittlich 3mal so hoch ermittelt worden.

Die mikroskopische Untersuchung des durch längeres Zentrifugieren von verdünnter Galle gebildeten Niederschlages hatte bei beiden Tieren dieselben Bilder geliefert:

Massen von Detritus, mehr oder weniger bräunlich gefärbt, darunter kristallinische gefärbte Gebilde, aber keine deutliche Zellen noch sonstige histologische Bestandteile.

\section{$\S$ 4. Bisherige Resultate von Gallenuntersuchungen nach Phosphor- und Arsenintoxikation.}

So ansehnlich die Zahl der experimentellen Arbeiten über die Wirkung des Arsens und des Phosphors auf die Leber auch ist, so hat sich die überwiegende Mehrzahl der Forscher mit den durch diese Gifte gesetzten Gewebsveränderungen beschäftigt und nur einer hat eine planvolle Erforschung des durch Phosphor und Arsen beeinflußten Lebersekrets nach Anlegung von Gallenfisteln an Hunden versucht, Stadelmann (Der Ikterus, S. 176 ff.)

Dem Phosphor schreibt Stadelmann einen spezifischen, die Sekretion anregenden Einfluß auf die Leberzellen zu; vermehrte Gallenfarbstoffausscheidung, Dunkelwerden der Galle ist die Folge.

Den Grund für die Vermehrung des Gallenfarbstoffs sieht Stadelmann mit Fränkel, Röhmann u. a. in der Zerstörung der roten Blutkörperchen durch den Phosphor. Der frei werdende Blutfarbstoff wird dann in der Leber zu Gallenfarbstoff umgewandelt. Ähnlich kann man gelegentlich bei Injektion von Hämoglobin in die Blutbahn eine Verminderung der Gallenmenge mit Vermehrung des Farbstoffs in derselben beobachten. - Während so im ersten Stadium der Phosphor die Bildung von Gallenfarbstoff begünstigt, folgt auf diẹ anfängliche Vermehrung bald ein 2. Stadium der Abnahme der Gallensekretion überhaupt. Gleichzeitig hat Stadelmann die Menge der ausgeschiedenen Gallensäuren eher vermindert als vermehrt gefunden, eine Tatsache, für die Brauer (Untersuchungen über die Leber, Diese Zeitschrift, Bd. XL, S. 186 ff. in dem Fortfall des intermediären Gallenkreislaufs der Gallen- 
säuren, in Ernährungsstörungen u. a. plausible Giünde gefunden hat. Zwischen beiden Stadien schiebt sich ein Zwischenraum von 2-3 mal 24 Stunden ein, in welchem an der Galle keine Abnormitäten sichtbar sind. Im 3. Stadium beginnt die Galle wieder klarer, dunkler, reicher an Farbstoff zu werden, während gleichzeitig der im 2. Stadium gesetzte Icterus der Gewebe abnimmt. Die Gallensäureausscheidung sinkt durch alle drei Stadien stetig.

Das Endresultat der Arsenversuche Stadelmanns stimmt mit dem der Phosphorversuche in den Hauptpunkten überein. Es stellte sich heraus, daß die Bilirubinausscheidung durch die Galle bis zum $3^{1 / 2}$ fachen des Normalen vermehrt war. Gleichzeitig fand aber eine Verminderung der Gallenquantität statt, die Galle wurde dickflüssiger, dunkler und außerordentlich zähe. Und ganz analog wie der Phosphor bewirkt auch die Arsenvergiftung eine sehr bedeutende Abnahme der ausgeschiedenen Gallensäure, so daß letztere bis auf $1 / 10$ des normalen Wertes heruntergehen kann.

\section{§ 5. Eigene Phosphorversuche.}

Nach Festlegung der normalen Gallenwerte für den Hund (Tab. I) wurde am 7. Juli mit der Phosphorintoxikation begonnen. Verf. bediente sich dazu der subkutanen Applikationsart, deren Vorzüge vor der Darreichung per os schon Aufrecht, Dinkler u. a. erörtert haben. Zur Anwendung gelangte eine möglichst gesättigte Lösung von Phosphor in Olivenöl. Hiervon wurde dem Hund zunächst $1 \mathrm{ccm}$ subkutan eingespritzt. Nachdem dieselbe Dosis im Zeitabstande von je $2 \mathrm{mal}$ 24. Stunden noch 2mal wiederholt war, wurde zur Steigerung der Giftwirkung die gleiche Dosis schon nach ca. 36 Stunden und nach wiederum 36 Stunden die 5 . Dosis gegeben. Der 6. Injektion, welche nach 24 Stunden folgte, erlag das Versuchstier.

In nachstehender Tabelle III sind die innerhalb der Zeit vom 7. bis 15. Juli gewonnenen Werte und Beobachtungen zusammengestellt.

Zunächst ersieht man aus unserer Tabelle, daß das subjektive Befinden des Tieres sich nicht sofort verschlechtert, sondern daß zunächst eine Zeit unveränderten Wohlbefindens folgt. Dieselbe hat bei uns bis zur 4. Injektion angehalten. Ein euphorisches Stadium, das nach dem anfänglichen Erbrechen 2-3 Tage anhält, wird auch nach Vergiftungsfällen bei Menschen beschrieben. 
Tabelle III.

\begin{tabular}{|c|c|c|c|c|c|c|c|c|}
\hline & 8. Juli & 9. Juli & 10. Juli & 13. Juli & 14. Juli & 15. Juli & 15. Juli & 16. Juli \\
\hline $\begin{array}{l}\text { Zeitraum } \\
\text { seit der } \\
\text { Injektion }\end{array}$ & $\begin{array}{l}16 \text { Stunden } \\
\text { nach der } \\
\text { crsten } \\
\text { Injektion }\end{array}$ & $\begin{array}{c}46 \text { Stunden } \\
\text { nach der } \\
\text { ersten } \\
\text { Injektion }\end{array}$ & \begin{tabular}{|} 
2.4 Stunden \\
nach der \\
zweiten \\
Injektion
\end{tabular} & $\mid \begin{array}{c}40 \text { Stunden } \\
\text { nach der } \\
\text { dritten } \\
\text { Injektion }\end{array}$ & $\begin{array}{l}20 \text { Stunden } \\
\text { nach der } \\
\text { vierten } \\
\text { Injektion }\end{array}$ & $\begin{array}{c}18 \text { Stunden } \\
\text { nach der } \\
\text { fünften } \\
\text { Injektion }\end{array}$ & $\begin{array}{l}23 \text { Stunden } \\
\text { nach der } \\
\text { fünften } \\
\text { Injektion }\end{array}$ & \\
\hline Befinden & $\begin{array}{l}\text { Unverändert } \\
\text { gut, munter, } \\
\text { Appetit gut }\end{array}$ & $\mid \begin{array}{c}\text { Gut, } \\
\text { Munterkeit, } \\
\text { Appetit } \\
\text { unverändert }\end{array}$ & $\begin{array}{l}\text { Wohl und } \\
\text { munter, } \\
\text { frist gut }\end{array}$ & $\begin{array}{c}\text { Unverändert } \\
\text { gut, } \\
\text { fribt gut }\end{array}$ & $\begin{array}{l}\text { Weniger } \\
\text { munter, } \\
\text { frißt } \\
\text { weniger }\end{array}$ & $\mid$\begin{tabular}{c|} 
Sichtlich \\
maiter, \\
magerer, \\
fribt nur \\
noch wenig
\end{tabular} & $\begin{array}{l}\text { Noch matter, } \\
\text { frift nicht }\end{array}$ & \multirow{12}{*}{ 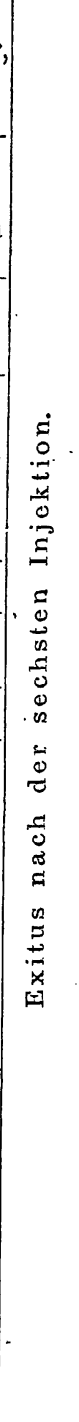 } \\
\hline $\begin{array}{c}\text { Ge- } \\
\text { wonnene } \\
\text { Gallen- } \\
\text { menge }\end{array}$ & $\begin{array}{c}30 \mathrm{ccm} \\
\text { in } 1 \frac{1}{2} \mathrm{St}\end{array}$ & $\left|\begin{array}{cc}30 & \mathrm{ccm} \\
\text { in } 1 / 2 & \text { St. }\end{array}\right|$ & $\begin{array}{c}35 \mathrm{ccm} \\
\text { in } 1 / 2 \mathrm{St} .\end{array}$ & $\mid \begin{array}{c}25 \mathrm{ccm} \\
\text { in } 1 \frac{1}{2} \mathrm{St} .\end{array}$ & $\begin{array}{c}22 \mathrm{ccm} \\
\text { in } 21 / 2 \mathrm{St} \text {. }\end{array}$ & $\begin{array}{r}3 \mathrm{ccm} \\
\text { in } 2 \mathrm{St} .\end{array}$ & $\begin{array}{c}5 \mathrm{ccm} \\
\text { in } 3 \text { Stunden }\end{array}$ & \\
\hline Farbe & $\begin{array}{l}\text { Wie früher } \\
\text { braun-grün }\end{array}$ & Dunkler & $\begin{array}{l}\text { Etwas heller } \\
\text { als gestern }\end{array}$ & $\begin{array}{c}\text { Dunkler, } \\
\text { undurch- } \\
\text { sichtig }\end{array}$ & $\begin{array}{c}\text { Sehr dunkel, } \\
\text { trüb }\end{array}$ & , Tief dunkel, & , Tief dunkel, & \\
\hline $\begin{array}{c}\text { Be- } \\
\text { schaffen- } \\
\text { heit }\end{array}$ & $\begin{array}{c}\text { Weder Blut } \\
\text { noch } \\
\text { Schleim } \\
\text { sichtbar }\end{array}$ & $\begin{array}{c}\text { Dick- } \\
\text { flüssiger, } \\
\text { ohne Bei- } \\
\text { mischungen }\end{array}$ & $\begin{array}{c}\text { Klar, ohne } \\
\text { Schleim } \\
\text { oder Blut }\end{array}$ & $\mid \begin{array}{l}\text { Sehr dick- } \\
\text { flüssig, } \\
\text { ohne Blut }\end{array}$ & \begin{tabular}{|} 
Dick, ohne \\
Schleim \\
noch Blut \\
$\vdots$
\end{tabular} & \begin{tabular}{|c|} 
Schmierig- \\
dick, durch \\
wiederholte \\
Blut- \\
beimischung \\
aus der Tiefe \\
der Leber \\
verunreinigt \\
\end{tabular} & $\begin{array}{c}\text { Dick- } \\
\text { schmierig, } \\
\text { aber ohne } \\
\text { Blut }\end{array}$ & \\
\hline Reaktion & Alkalisch & Alkalisch & Alkalisch & Alkalisch & Alkalisch & Alkalisch & Alkalisch & \\
\hline $\begin{array}{c}\text { Spezif. } \\
\text { Gewicht } \\
\end{array}$ & 1027 & 1031 & 1020 & 1037 & 1026 & - & 1029 & \\
\hline Viskosität & 40,8 & 56 & 32,9 & 73 & 55,1 & - & 62,8 & \\
\hline $\begin{array}{l}\text { Koch- } \\
\text { probe }\end{array}$ & $\begin{array}{c}\text { Deutliche } \\
\text { Trübung, } \\
\text { nach Stehen } \\
\text { deutlicher } \\
\text { Bodensatz }\end{array}$ & $\begin{array}{l}\text { Deutlich } \\
\text { positiv }\end{array}$ & $\begin{array}{l}\text { Nur Spur } \\
\text { Opaleszenz }\end{array}$ & \begin{tabular}{|c|} 
Deutlicher \\
flockiger \\
Nieder- \\
schlag \\
\end{tabular} & $\begin{array}{c}\text { Kolossale } \\
\text { Trübung } \\
\text { und Aus- } \\
\text { scheidung } \\
\text { auf } \\
\text { dem Boden } \\
\end{array}$ & - & Stark positiv & \\
\hline $\begin{array}{l}\text { Zucker- } \\
\text { probe }\end{array}$ & Negativ & Negativ & Negativ & Negativ & Negativ & - & - & \\
\hline $\begin{array}{l}\text { Gesamt-N } \\
\text { in } 5 \mathrm{ccm}\end{array}$ & $13,30 \mathrm{mg}$ & $20,58 \mathrm{mg}$ & $10,50 \mathrm{mg}$ & $25,76 \mathrm{mg}$ & $16,52 \mathrm{mg}$ & - & - & \\
\hline $\begin{array}{l}N \text { des } \\
\text { Alkohol- } \\
\text { nieder- } \\
\text { schlags } \\
\text { in } 5 \mathrm{ccm}\end{array}$ & $2,38 \mathrm{mg}$ & $6,02 \mathrm{mg}$ & $1,4 \mathrm{mg}$ & $4,48 \mathrm{mg}$ & $5,88 \mathrm{mg}$ & - & - & \\
\hline $\begin{array}{l}\text { Mikrosk. } \\
\text { Befund }\end{array}$ & $\begin{array}{c}\text { NichtsHisto- } \\
\text { logisches, } \\
\text { nurDetritus, } \\
\text { Kristalle u. } \\
\text { Farbstoff } \\
\text { wie früher }\end{array}$ & $\begin{array}{c}\text { Sehr viel } \\
\text { Fett- } \\
\text { Tröpfchen, } \\
\text { körniger } \\
\text { Detritus } \\
\end{array}$ & $\begin{array}{l}\text { Wie tags } \\
\text { zuvor, } \\
\text { insbeson- } \\
\text { dere keine } \\
\text { Zylinder }\end{array}$ & \begin{tabular}{|c|} 
Fett- \\
kügelchen, \\
körniger \\
Detritus mit \\
Gallenfarb- \\
stoff, verein- \\
zelte körnig \\
degenericrte \\
Leberzellen; \\
doch keine \\
Zylinder
\end{tabular} & $\begin{array}{l}\text { Derselbe } \\
\text { Befund } \\
\text { wie } \\
\text { gestern }\end{array}$ & e & $\begin{array}{c}\text { Viel Detritus, } \\
\text { weniger Fett, } \\
\text { viel Farbstoff, } \\
\text { einige schlauch- } \\
\text { artige Zylinder } \\
\text { mit Andeutung } \\
\text { von Dichotomie- } \\
\text { Leberzellen }\end{array}$ & \\
\hline
\end{tabular}


Sehr deutlich zeigen unsere Werte das erste der Stadelmannschen Stadien bei Phosphorintoxikation, das durch Vermehrung der sekretorischen Tätigkeit der Leberzellen charakterisiert ist. Zwar bewegten sich unsere normalen Bestimmungen (Tab. I) an den letzten 3 Tagen schon in recht hohen Werten - am 6. VII. waren es $50 \mathrm{ccm}$ in 1 Stunde doch werden diese Zahlen noch übertroffen von den Werten am 9. und 10. Juli, an welchen unter der Wirkung der 2 ersten Dosen Phosphoröl 30 resp. $35 \mathrm{ccm}$ Galle in 1/2 Stunde gewonnen wurden. Nach der 3. Injektion beginnt das 2. Stadium, das rasche Absinken der Absonderungsgeschwindigkeit. Freilich wird man neben der gesteigerten Giftwirkung auch die verminderte und schließlich ganz aussetzende Freßlust des Tieres hierfür verantwortlich zu machen haben. Mit der Wirkung der 5. Injektion tritt schon ein fast gänzliches Versagen des Gallenabflusses ein. Dafür fließt jetzt eine blutähnliche braunrote Flüssigkeit aus der Lebertiefe, die sich durch relativ dunklere Farbe und ihre Dickflüssigkeit deutlich von normalem Blut unterscheidet. Stadelmann, der ähnliches beobachtet, weist den Gedanken, daß es sich um beginnende Hämoglobinocholie handle, zurück und sieht die Ursache in einer Läsion der Fistelwand. Andere wie Filehne wollen Hämoglobinocholie nach Applikationen von blutauflösenden Mitteln häufig bei Kaninchen, nicht aber bei Hunden gesehen haben. Wertheimer und Meyer bezeichnen Hämoglobinocholie selbst nach intravenösen Injektionen großer Mengen von Hämoglobin, denen starke Hämoglobinurie folgte, als eine Seltenheit.

Die mikroskopische Untersuchung des Sekrets ergab neben intakten roten Blutkörperchen zahlreiche Ponficksche Schatten. $\mathrm{Zu}$ einer spektroskopischen Untersuchung kam es infolge eintretenden exitus des Tieres nicht, so daß wir die Entscheidung der Frage offen lassen müssen.

Die Farbe der Galle wurde schon nach der ersten Injektion dunkler; jedoch begann die Farbstoffbildung nicht sofort, sie war 16 Stunden nach der Injektion noch nicht wahrzunehmen; wohl aber schon ganz ausgesprochen nach weiteren 20 Stunden. Eine Sonderstellung nimmt sowohl hier als auch 
hinsichtlich aller übrigen Bestimmungen der 10. Juli ein; mit seinen dem Normalen nahestehenden Werten würde er der zwischen 1. und 2. Stadium liegenden «intermediären Pause» Stadelmanns entsprechen.

Diese intermediäre Pause lagert er (S. 249) vor dem Absinken der anfänglich gesteigerten Gallenabsonderung, was mit unserer Beobachtung stimmt.

Um dieselbe Zeit soll nach Stadelmann auch die bedeutend vermehrte Absonderung zähen Schleims einsetzen; auch diese Beobachtung können wir bestätigen, aber unsere Versuche zeigen auch, daß derselbe im wesentlichen den letzten Strecken der Ausführungswege entstammt und nicht so sehr den mehr zentralern; denn er floß nicht durch die Kanüle ab, sondern umgab den Ausgang der Fistelöffnung. Auf dasselbe Verhalten weisen auch unsere viskosimetrischen Messungen hin; dieselben zoigen keine wesentliche Erhöhung der Werte gegenüber den Normalbestimmungen. ${ }^{1}$ )

Vergleichen wir die Werte für das spezifische Gewicht unserer Tabelle mit denen der zugehörigen Tabelle I, so ist eine leichte Erhöhung derselben zu erkennen.

Vielleicht das interessanteste Ergebnis lieferten die Proben auf koagulierbares Eiweiß. Gleich nach der ersten Injektion stellte sich eine so starke Albuminocholie ein, daß eine ganz deutliche intensive Trübung der vorher durchsichtig klaren Galle entstand. Nach kurzem Stehen lag diese Eiweißfällung als deutlicher Niederschlag auf dem Boden. Dieses positive Ergebnis

1) Freilich soll nicht geleugnet werden, daß die Phosphorvergiftung auch einen Katarrh der feineren Gallenwege zustande bringt. Ein Katarrh der makroskopisch sichtbaren feinen Gallengänge ist von W. Ebstein schon 1868 an menschlichen Phosphorleichen beschrieben und der gleichzeitig vorhandene Icterus hierdurch erklärt werden. (Archiv für Heilkunde, 9. Jahrg., S. 219.) Und schon vorher haben Wyss und Alter dieselben Tatsachen experimentell an Hunden festgestellt. - Angesichts unserer obigen Bemerkungen erhebt sich jedoch die Frage, ob man wirklich berechtigt ist, den Grund des Icterus bei Phosphorvergiftung in einer Resorption der wegen ihrer Zähigkeit nicht abfließenden pleiochromen Galle zu erblicken. Jedenfalls wären viskosimetrische Messungen hier sehr zu wünschen. 
der Eiweißprobe wurde mit jedem Tage deutlicher - mit Ausnahme des als "intermediäre Pause» aufzufassenden 10. Juli. Abgesehen von dieser Besonderheit verhält sich also die Schädigung der Leberfunktion durch Phosphor genau so wie die durch Alkohol: es tritt sehr frühe eine Eiweißabscheidung und Abfuhr desselben mit dem Lebersekret ein.

Die Zahlen, welche die Kjeldahl-Bestimmungen ergeben haben, zeigen für den Gesamtstickstoff eine Zunahme auf fast das Doppelte des normalen Betrags. Mit fortschreitender Intoxikation scheint jedoch dieser Betrag wieder abzusinken, doch schloß die versiegende Gallensekretion der zwei letzten Versuchstage weitere Bestimmungen des Gesamtstickstoffs aus. Die Gründe für die gefundene Stickstoffverdoppelung können nur zum geringsten Teil in der Farbstoffvermehrung liegen, die bekanntlich stets nur einen geringen Anteil der festen Bestandteile der Galle ausmachen und einer Steigerung nur im Rahmen von $0,6-0,7 \%$ bis 1,3\% der festen Bestandteile fähig sind. Auch durch das Hinzukommen von Eiweiß allein läßt sich dies N-Anwachsen nicht erklären. Ein Blick auf die Tabelle genügt, um zu ersehen, daß zwischen den Zahlen für Gesamt-N und Stickstoff des Alkoholniederschlags nicht einmal Parallelität besteht.

Für die Urinsekretion, welche ebenfalls eine deutliche Vermehrung des Gesamtstickstoffs bei Phosphorvergiftung zeigt, ist es ja nachgewiesen, daß ein nicht unbeträchtlicher Teil des Stickstoffs den Körper als Ammoniak verläßt. Wie man sich für die Gallensekretion ein Analagon dieses Verhaltens des Stickstoffs zu denken hat, läßt sich zunächst nicht absehen.

Die mikroskopische Untersuchung zeigte nach der 3. Injektion zum erstenmal deutlich histologische Elemente, die in Form und Größe auf trübkörnig degenerierte Leberzellen hinwiesen. Endlich wenige Stunden vor dem exitus gelang es, einzelne Schläuche festzustellen, von denen die einen mehr homogen waren, nur einige wenige Kerne regellos verteilt enthielten, andere dagegen Schollen einschlossen und dichotomische Teilung zeigten, während wiederum Kerne nur in kleiner Anzahl darin lagen. Diese Bildungen entsprachen also einigermaßen den Brauerschen Zylindern, wie sie dieser bei seinen 
Alkoholversuchen und Haup t lbei 'Schwefelkohlenstoffvergiftun gefunden und beschrieben hal (ef. Brauer, a. a. 0., S. 213 f.) Wir schließen diesen Absschnitt, indem wir das Ergebnis der Sektion, soweit es hier won Interesse ist, anfügen. Das Tier wurde in einer Lache wenig oder gar nicht geronnenen dunkelfarbigen Blutes tot aufgrefunden. Aus Mund und Nase waren größere stattgebabte Blutungen zu erkennen, ebenso aus der Fistelöffnung. Die Gewebe: zeigten keinen ausgeprägten Icterus. Die Leber bot das typische Bild der Phosphorleber, sie war etwas vergrößert, von weicher Konsistenz, sehr blutreich. Viele parenchymatöse Blutungien sind sichtbar; Zeichnung der Acini sehr deutlich, die Zellen feltig infiltriert. Nieren etwas geschwollen, Herzmuskel makrioskopisch nicht deutlich verfettet. Die Pleuren und das Peritoneum zeigen eine große Anzahl verschiedener großer, petechialer und größerer Blutungen. Das im Körper enthaltene Blut gerinnt nicht. Die mikroskopisch untersuchten Leberteile ergaben eine ganz auffallend starke Veränderung des Leberparenchyms. Die innere Struktur der Acini war fast ganz verloren gegangen, von Zellbalkenreihen nichts mehr zu unterscheiden; überall eín regelloses Gewirr von mehr weniger degenerierten polygonalen Zellen mit teilweise schlecht färbbarem Kern, trübem Protoplasma und feinkörniger Fettinfiltration. Stellenweise starke vacuoläre Degeneration. Blutkörperchen reichlich zwischen den Zellmassen. Eine Gallengangswucherung ist nirgends erkennbar, ebenso keine Bindegewebsvermehrung. Alle sichtbaren Gallengänge - sowohl auf Quer-, wie Schräg-, wie Längsschnitten - sind erfüllt mit einer homogenen Masse, die sich als Schleim erweist und in welcher Kerne eingelagert sind. Auch regelmäßige Nebeneinanderordnungen von mehreren Kernen - an einer Stelle waren es 5 - kommen vor. Doch unterscheiden sich die Kerne deutlich von den Kernen der Wandepithelien nach Größe und Färbung. Die Gallengangswände sind anscheinend tiberall gut erhalten, desquamative Prozesse sind nirgends sicher festzustellen.

$\S 6$. Eigene Arsenversuche.

Zur Anwendung kam die namentlich in subkutaner Applikation sehr bequem zu dosierende Fowlersche Lösung. 
Am 14. Juli bekam die Hiünrdin als erste Injektion $0,03 \mathrm{~g}$ Liquor Kali arsenicosi. Nachderm die gleiche Dosis am nächsten Tage wiederholt war, wurde die 3 . Injektion auf 4 Pravazspritzen gesteigert. Am nächstfolgendem Tage fand gelegentlich der $\dot{4}$. Injektion wiederum eine Steiggerung auf $0,05 \mathrm{~g}$ statt und in gleicher Weise jeden folgenden Tag, mit Ausnahme des 19. Juli, an welchem gar keine Injektion gemacht wurde, bis mit der 7. Injektion die Dosis 0,08 $\mathrm{g}$ Kali arsen, erreicht war. Da der Zustand des Tieres, insbetsondere die multiplen, handtellergroßen Hautnekrosen zu einer raschen Beendigung der Versuche trieben, so wurde die 8. Injektion auf $0,12 \mathrm{~g}$ gesteigert. Erst der 9. Injektion von $0,20 \mathrm{~g}$ erlag das Tier.

Wir lassen wiederum zunächst eine tabellarische Übersicht der gewonnenen Werte folgen.

Hier wie bei den Phosphorversuchen zeigte sich das subjektive Befinden des Versuchstieres nicht sofort alteriert. Dagegen stellten sich zum Schluß außer den schon erwähnten Hautnekrosen sehr schwere Störungen ein, die sich bis zur Parese der hinteren Extremitäten steigerten.

Die von Stadelmann nach Arsen konstatierte Abnahme der Gallenquantität tritt bei unseren Versuchen erst in den letzten 2 Tagen in die Erscheinung, d. h. zu einer Zeit, wo das Tier zu hungern begann. Die Wirkung des Arsens auf das Quantum der Gallensekretion ist also hier ausgeblieben oder doch nicht deutlich.

Die Farbenbestimmungen zeigen im Gegensatz zu denen der Phosphorversuche ein Hellerwerden der Galle, während erst gegen das Ende zu der Gallenfarbstoff zunimmt. Dabei war aber bis zum Ende der braune Farbenton der Galle gegenüber dem grünen bedeutend vorherrschend, während nach Phosphor sehr früh die grüne gegenüber der braunen Komponente sich im Übergewicht befand. Möglicherweise ist hierin ein Hinweis auf die oxydierende Wirkung des Phosphors gegeben; stellt doch das Biliverdin eine Oxydationsstufe des Bilirubins dar.

Im allgemeinen zeigte sich überall eine Parallelität der Konsistenz der Galle mit dem Farbenton dergestalt, daß die hellere Galle zugleich auch die dünnflüssigere, die dunklere zugleich auch die dickere war. 
Tabelle IV.

\begin{tabular}{|c|c|c|c|c|c|c|c|c|c|}
\hline & 15. Juli & 16. Juli & 17. Juli & 18. Juli & 20. Juli & 21. Juli & 22. Juli & 23. Juali & 23.Jati \\
\hline $\begin{array}{c}\text { Zeit- } \\
\text { dauer } \\
\text { seit der } \\
\text { In- } \\
\text { jektion }\end{array}$ & $\left|\begin{array}{c}22 \text { Std. } \\
\text { nach der } \\
\text { 1. In. } \\
\text { jektion }\end{array}\right|$ & $\begin{array}{c}14 \text { Std. } \\
\text { nach der } \\
\text { 2. Injektion }\end{array}$ & $\begin{array}{c}15 \text { Std. } \\
\text { nach der } \\
\text { 3. Injektion }\end{array}$ & $\begin{array}{c}15 \mathrm{Std} . \\
\text { nach der } \\
\text { 4. Injoktion }\end{array}$ & $\begin{array}{c}40 \text { Std. } \\
\text { nach der } \\
\text { 5. Injektion }\end{array}$ & $\begin{array}{c}22 \text { Std. } \\
\text { nach der } \\
\text { 6. Injektion }\end{array}$ & $\begin{array}{c}22 \text { Std. } \\
\text { nach der } \\
\text { 7. Injektion }\end{array}$ & $\begin{array}{c}20 \text { Std. } \\
\text { nach der } \\
\text { 8. Injektion }\end{array}$ & $\begin{array}{c}\text { 5 Stdd } \\
\text { n. d.9. } \\
\text { Injek- } \\
\text { tion }\end{array}$ \\
\hline $\begin{array}{c}\mathrm{Be}- \\
\text { finden }\end{array}$ & \begin{tabular}{|c|} 
Unver- \\
ändert \\
große \\
Freß- \\
lust \\
Gew. \\
$17,5 \mathrm{~kg}$ \\
\end{tabular} & \begin{tabular}{|l|} 
Mratter, doch \\
Freßlust un- \\
vermindert
\end{tabular} & $\left|\begin{array}{c}\text { Unverändert } \\
\text { munter, frißt } \\
\text { gierig } \\
\text { Gew. } 17,5 \mathrm{~kg}\end{array}\right|$ & $\begin{array}{c}\text { Etwas } \\
\text { matter, frißt } \\
\text { unvermin- } \\
\text { dert }\end{array}$ & \begin{tabular}{|} 
Gut, munter, \\
fribt gut, \\
Hautnekro- \\
sen beginnen \\
Gerv. $18,0 \mathrm{~kg}$
\end{tabular} & $\begin{array}{l}\text { Matt, krank, } \\
\text { Hautaffekte } \\
\text { vergrößert, } \\
\text { sezernieren } \\
\text { stark. } \\
\text { Frißt gut }\end{array}$ & \begin{tabular}{|l|} 
Verschlech- \\
tert, Nekro- \\
sen fortge- \\
schritten. \\
FriBt kaum \\
Gew. 17,5 kg
\end{tabular} & \begin{tabular}{|c|} 
Bedeutend \\
verschlech-| \\
tert, hin- \\
fällig, frißt \\
nicht, unru- \\
hig, heult \\
laut
\end{tabular} & $\frac{\ddot{z}}{\bar{z}}$ \\
\hline $\begin{array}{l}\text { Gallen- } \\
\text { menge }\end{array}$ & $\mid \begin{array}{c}\text { wenige } \\
\text { Tropfen } \\
\text { in 3 St. }\end{array}$ & in $1 \frac{\mathrm{ccm}}{2} \mathrm{~s}$ St. & $\left|\begin{array}{cc}25 \mathrm{ccm} \\
\text { in } 21 / 2 & \mathrm{St}\end{array}\right|$ & $\left|\begin{array}{cc}25 & \mathrm{ccm} \\
\text { in } 11 / 4 & \mathrm{St}\end{array}\right|$ & $\begin{array}{c}25 \mathrm{ccm} \\
\text { in } 1 \frac{1}{2} \mathrm{St}\end{array}$ & $\begin{array}{c}20 \mathrm{ccm} \\
\text { in } 13 / 4 \mathrm{St}\end{array}$ & $\begin{array}{l}18 \mathrm{ccm} \\
\text { in } 3 \mathrm{St} .\end{array}$ & $\begin{array}{l}\text { wenige } \mathrm{ccm} \\
\text { in } 3 \mathrm{St} \text {. }\end{array}$ & - \\
\hline $\begin{array}{c}\text { Farbe } \\
\text { der- } \\
\text { selben }\end{array}$ & $\begin{array}{l}\text { Hell } \\
\text { klar }\end{array}$ & $\begin{array}{c}\text { Weniger } \\
\text { hell, mehr } \\
\text { grünbraun, } \\
\text { klar }\end{array}$ & \begin{tabular}{|c|} 
Anfangs \\
dunkelbraun- \\
grün, dann \\
heller, klar
\end{tabular} & $\begin{array}{c}\text { Auffallend } \\
\text { hell, kaum } \\
\text { grünlich; } \\
\text { klar }\end{array}$ & \begin{tabular}{|} 
Ganz auf- \\
fallend hell, \\
bernstein- \\
farbig; klar
\end{tabular} & $\begin{array}{c}\text { Weniger } \\
\text { hell, vor- } \\
\text { wiegend } \\
\text { braun }\end{array}$ & $\begin{array}{c}\text { Etwas } \\
\text { dunkler }\end{array}$ & $\left|\begin{array}{c}\text { tief dunkel- } \\
\text { braun } \\
\text { (wenig grün) }\end{array}\right|$ & - \\
\hline $\begin{array}{c}\text { Sonstigo } \\
\text { Be- } \\
\text { schaffen- } \\
\text { heil }\end{array}$ & $\begin{array}{c}\text { Relativ } \\
\text { dünn- } \\
\text { flüssig, } \\
\text { kein } \\
\text { Blut } \\
\text { noch } \\
\text { Schleim } \\
\text { sichthar }\end{array}$ & $\begin{array}{c}\text { Kaum } \\
\text { dicker, frei } \\
\text { von Blut } \\
\text { und } \\
\text { Schleim }\end{array}$ & \begin{tabular}{|c|} 
Anfangs \\
dick, faden- \\
ziehend, \\
dann \\
wesentlich \\
dünner. Kein \\
Blut noch \\
Schleim \\
\end{tabular} & $\begin{array}{c}\text { Dünn, frei } \\
\text { von Blut } \\
\text { und. } \\
\text { Schleim }\end{array}$ & $\begin{array}{c}\text { Sehr dünn- } \\
\text { flüssig. Kein } \\
\text { Blut noch } \\
\text { Schleim }\end{array}$ & $\begin{array}{c}\text { Weniger } \\
\text { dünn, kein } \\
\text { Blut noch } \\
\text { Schleim }\end{array}$ & \begin{tabular}{|c|} 
Etwas \\
dicker, \\
namentlich \\
zuletzt; ohne \\
Blut und \\
Schleim
\end{tabular} & $\begin{array}{c}\text { Schmierig- } \\
\text { dick, faden- } \\
\text { ziehend, } \\
\text { trübe }\end{array}$ & \\
\hline Reaktion & Alkal. & Alkalisch & Alkalisch & Alkalisch & Stark alkal. & Alkalisch & Alkalisch & Alkalisch & - \\
\hline Spez. Gerr. & - & 1022 & 1019 & 1016 & 1017 & 1018 & 1018 & 1026 & - \\
\hline Tiskosität & - & 37,1 & 32,4 & 32,3 & 28,8 & 33,5 & 35,7 & $117^{\circ}$ & - \\
\hline $\begin{array}{c}\text { Probe anf } \\
\text { kosgolier- } \\
\text { bares } \\
\text { Birreils in } \\
\text { der Galle }\end{array}$ & \begin{tabular}{|c|} 
Deut- \\
liche \\
Trü- \\
bung, \\
fein- \\
flockiger \\
Nieder- \\
schlag
\end{tabular} & $\begin{array}{c}\text { Starke } \\
\text { Trübung } \\
\text { und starker } \\
\text { flockiger } \\
\text { Nieder- } \\
\text { schlag }\end{array}$ & \begin{tabular}{|c|} 
Starke \\
Trübung, \\
beträcht- \\
licher \\
Bodensatz \\
beim Stehen
\end{tabular} & $\begin{array}{c}\text { Trübung } \\
\text { und Boden- } \\
\text { satz nach } \\
\text { Stehen }\end{array}$ & $\begin{array}{c}\text { Schwache } \\
\text { Trübung, } \\
\text { Bodensatz } \\
\text { nach Stehen }\end{array}$ & $\begin{array}{l}\text { Deutliche } \\
\text { Trübung, } \\
\text { flockiger } \\
\text { Nieder- } \\
\text { schlag }\end{array}$ & $\begin{array}{c}\text { Erhebliche } \\
\text { Trübung, } \\
\text { klein- } \\
\text { flockige } \\
\text { Fällung }\end{array}$ & $\begin{array}{c}\text { Ganz dicke } \\
\text { Fällung } \\
\text { noch in } \\
\text { starker Ver- } \\
\text { dünnung }\end{array}$ & - \\
\hline $\begin{array}{c}\text { Eiweiß- } \\
\text { probe } \\
\text { im Harn }\end{array}$ & Negativ & Negativ & Zweifelhaft & $\begin{array}{c}\text { Schwach } \\
\text { positiv }\end{array}$ & $\begin{array}{c}\text { Schwach } \\
\text { positiv }\end{array}$ & $\begin{array}{c}\text { Deutlich } \\
\text { positiv }\end{array}$ & $\begin{array}{c}\text { Deutlich } \\
\text { positiv }\end{array}$ & - & $\begin{array}{l}\text { Posi- } \\
\text { tiv }\end{array}$ \\
\hline $\begin{array}{c}\text { Zucker- } \\
\text { probe }\end{array}$ & - & Negativ & Negativ & Negativ & Negativ & Negativ & Negativ & Negativ & - \\
\hline $\begin{array}{r}\text { Ges.-N } \\
\text { in } 5 \mathrm{ccm}\end{array}$ & - & $12,6 \mathrm{mg}$ & $9,52 \mathrm{mg}$ & $7,42 \mathrm{mg}$ & $9,10 \mathrm{mg}$ & $10,64 \mathrm{mg}$ & $11,48 \mathrm{mg}$ & $13,86 \mathrm{mg}$ & - \\
\hline $\begin{array}{c}\text { N des } \\
\text { Alkohol- } \\
\text { Nieder- } \\
\text { schlags } \\
\text { in } 5 \text { ccm }\end{array}$ & - & $3,08 \mathrm{mg}$ & $2,38 \mathrm{mg}$ & $1,26 \mathrm{mg}$ & $1,96 \mathrm{mg}$ & $2,24 \mathrm{mg}$ & $2,52 \mathrm{mg}$ & $3,78 \mathrm{mg}$ & - \\
\hline $\begin{array}{l}\text { Mi- } \\
\text { kro- } \\
\text { skop. } \\
\text { Be- } \\
\text { fund }\end{array}$ & - & $\begin{array}{c}\text { Viel } \\
\text { amorphe } \\
\text { Massen, } \\
\text { teilweise } \\
\text { schlauch- } \\
\text { artig ange- } \\
\text { ordnet, } \\
\text { einige Kri- } \\
\text { stalle, keine } \\
\text { Zellen noch } \\
\text { Zylinder }\end{array}$ & \begin{tabular}{|} 
Wenig Fett- \\
tröpfchen, \\
amorphe u. \\
kristalli- \\
nische \\
Massen, \\
durch Farb- \\
stoff gelb \\
tingiert, \\
keine deut- \\
liche histo- \\
logische Be- \\
standteile
\end{tabular} & \begin{tabular}{|} 
Einige kleine \\
Fett- \\
tröpfchen, \\
viel amorphe \\
körnige \\
Masse.Deut- \\
liche Leber- \\
zellen mit \\
körnig trü- \\
bem Proto- \\
plasma und \\
Fett gefüllt. \\
Schlätuche, \\
Kristalle
\end{tabular} & $\begin{array}{l}\text { Viel klein- } \\
\text { körnige } \\
\text { amorphe } \\
\text { zusammen- } \\
\text { geballte } \\
\text { Massen, in } \\
\text { denen } \\
\text { Leberzellen } \\
\text { liegen. Ein- } \\
\text { zelne Zylin- } \\
\text { der, weniger } \\
\text { homogene } \\
\text { als körnige }\end{array}$ & \begin{tabular}{|c|} 
Viel körnige, \\
einzelne \\
mehr homo- \\
gene Zylin- \\
der; Leber- \\
zellen deut- \\
lich aufzu- \\
finden. \\
Wenig \\
kleinste \\
Fett- \\
tröpfchen
\end{tabular} & \begin{tabular}{|c|} 
Deutliche \\
Zylinder, \\
fettig infil- \\
trierte \\
Leberzellen, \\
kleinste \\
Fett- \\
tröpfchen in \\
vermehrter \\
Zahl. Danc- \\
ben körniger \\
Detritus, \\
Schollen, \\
Kristalle
\end{tabular} & \begin{tabular}{|c|} 
Viel \\
Zylinder, \\
vereinzelte \\
große Zellen, \\
Leberzellen, \\
kleinkörni- \\
ges Material, \\
Fett- \\
tröpfchen, \\
vicl \\
Farbstoff \\
\end{tabular} & - \\
\hline
\end{tabular}


Damit stehen die Viskositätsbestimmungen im Einklang; sie zeigen im übrigen kein großes Anwachsen unter der Arsenwirkung gegenüber den normalen Werten der zugehörigen Tabelle II. - Nur der Wert vom Todestage fällt mit fast dem 4fachen Betrage der vorherigen Bestimmungen ganz plötzlich aus der Reihe und beweist die tiefgreifende Veränderung der Gallensekretion in den letzten Stunden vor dem Tode, Veränderungen, die wohl in dem massenhaften Übertritt von Zerfallsprodukten - Zellenmaterial und Fett vor allem - neben vermehrtem Farbstoff ihren Grund haben. Vermehrte Schleimabsonderung war nicht $\mathrm{zu}$ beobachten.

Die Zahlen für das spezifische Gewicht verhalten sich wie die der Viskosität: sie weichen von den Normalwerten desselben Tieres nicht allzuviel $\mathrm{ab}$ und zeigen eine genügende Konstanz.

In einer Hinsicht gleicht die Arsenwirkung auf die Galle gänzlich der des Phosphors: beide bewirken sofort deutliche Albuminocholie. Gleich die erste Gallenprobe ergibt beim Kochen deutliche Trübung und feinflockigen Niederschlag; zu dieser Zeit erschien die Galle weder nach Farbe noch nach Konsistenz irgendwie beeinflußt. Von da ab hat die Menge des ausgefällten Eiweißes stetig zugenommen. ${ }^{1}$ )

Die Untersuchung des Harns zeigte nach zwei Injektionen noch keine Albuminurie, auch nach der dritten Injektion war das Resultat noch zweifelhaft und erst die vierte Injektion ließ deutliche Trübung erkennen. Endlich erst nach der sechsten Injektion stellte sich ganz deutliche Eiweißausscheidung durch den Harn ein. Wir haben also bereits starke Albuminocholie $\mathrm{zu}$ einer Zeit konstatiert, zu welcher eine Albuminurie noch nicht eingetreten war. ${ }^{2}$ )

1) Die etwas schwächere Trübung am 20. Juli erklärt sich einmal aus der besonders starken Dünnflüssigkeit des Sekrets an diesem Tage; vielleicht auch aus dem Abstand von 40 Stunden seit der letzten Injektion, der eine vorübergehende Erholung des Versuchstieres ermöglichte.

2) Bei Untersuchungen über febrile Albuminurie hat Hallauer (Verhandl. der physikal.-mediz. Gesellschaft z. Würzburg 1903) gefunden, daß die pathologischen Veränderungen, welche bei der Niere die Eiweißausscheidung durch den Harn bedingen, in entsprechender Weise auch bei der Leber Übertritt von Eiweiß in die Galle bewirken. 
Die Zuckerproben waren auch hier, wie zu erwarten, gänzlich negativ.

Überblicken wir den Gang der Stickstoffbestimmungen, so zeigt unsere Versuchsreihe folgendes Verhalten: Gleich die erste Bestimmnng - nach der zweiten Injektion - ergibt ein Ansteigen des Gesamtstickstoffs fast auf das Doppelte des letzten normalen Wertes. Und gleichzeitig wächst der Stickstoff des Alkoholniederschlages auf mehr als das Dreifache des normaliter Gefundenen an. Dann aber gehen unter der Wirkung der dritten und vierten Injektion beide Werte bis zum Normalwerte herab. Von da ab findet ein Ansteigen statt, sodaß am Tage des Todes des Versuchstieres Zahlen gefunden werden, welche die hohen anfänglichen noch übertreffen.

Wenn man Stadelmanns Angaben über den Anteil der Farbstoffe und der Gallensäure in der Galle nach Arsenvergiftung heranzieht, so müßte man sich vorstellen, daß die anfänglich hohe Zahl für den Gesamtstickstoff erzielt ist, zu einer Zeit, wo die Eiweißabscheidung ein Plus von Eiweiß der Galle zuführt, wo aber das Gallensäuredefizit noch nicht begonnen hatte. Bilirubinvermehrung im Sinne Stadelmanns noch außerdem heranzuziehen, verbieten unsere Protokolle, die Galle wurde nicht dunkler, sondern eher heller. Nach der nächsten Injektion beginnt mit zunehmender Giftwirkung die bekanntlich bei Arsen besonders starke Abnahme der Gallensäure, während gleichzeitig ein Plus von Gallenstoffen - wie unsere Protokolle besagen - noch nicht eingetreten ist. Daher geht die Stickstoffausscheidung durch die Galle herab. Endlich wird das durch die fehlenden Gallensäuren geschaffene Defizit durch vermehrte Bilirubinausscheidung ausgeglichen; die Eiweißausscheidung und damit zugleich die des Gesamtstickstoffs steigt wieder an. Ob diese Darlegung zur Erklärung der Zahlen unserer Tabelle genügt, ist fraglich; eine andere steht aber bei unseren bisherigen Kenntnissen nicht zur Verfügung. Insbesondere sind die Stoffwechseluntersuchungen bei Arsenvergiftung in ihren Resultaten $\mathrm{zu}$ unsicher und einander widersprechend, als daß sie hier mit Nutzen heranzuziehen wären. 
$\mathrm{Zu}$ betonen ist, daß die Reihe der Werte für den Gesamtstickstoff hier eine völlige Parallelität mit den Bestimmungen des Stickstoffs des Alkoholniederschlages zeigt. Doch auch hier läßt sich ebenso wie bei den Phosphorversuchen das Anwachsen des Gesamtstickstoffs aus dem des steigenden $\mathrm{N}$ im Alkoholniederschlag nicht völlig herausrechnen.

Unsere mikroskopischen Befunde ergeben bei einem Vergleich mit denen nach Phosphor, daß nach Arsen das Fett später, histologische Bestandteile aber etwas früher und zahlreicher in der Galle auftreten. Schon nach der vierten Injektion zeigten sich viele Leberzellen, deren Protoplasma körnig getrübt und mit kleinsten Fettkügelchen erfüllt ist. Am gleichen Tage sind aber schon die bei unseren Phosphorversuchen erst am Todestage erscheinenden Brauerschen Zylinder zu finden und von da $a b$ hat Verfasser sie regelmäßig in jeder Gallenprobe vorgefunden. Einzelne derselben zeigten einen mehr homogenen fast hyalinen Bau, doch herrschten solche vor, die aus mehrkörnigen zusammengeballten Massen bestanden. Auch vereinzelte Epithelien und Zellikerne hatte Verfasser zu sehen Gelegenheit.

Das Ergebnis der Sektion der Hündin, die gleich nach eingetretenem Tode ausgeführt wurde, war kurz folgendes:

Die Haut zeigt an verschiedenen Stellen größere nekrotische, in Abstoßung begriffene Teile, an zwei Stellen sind dieselben mehr als handtellergroß. Starker Haarausfall.

Ausgesprochener Hydrops anasarka, kein Ascites. Blutgefäße strotzend völl dunklen, geronnenen Blutes.

Die Leber ist kaum vergrößert, dunkel braunrot, sehr morsch, stark hyperämisch. Acini gut abgegrenzt sichtbar, mit deutlicher Fettinfiltration.

Herzmuskel normal, Nieren sehr prall, Zeichnung der Harnkanälchen sehr deutlich, auch hier Hyperämie, jedoch geringer als in der Leber.

Der mikroskopische Leberbefund zeigt die Leberbalken schön erhalten, durch Hyperämie auseinandergedrängt, dazwischen viel rote Blutkörperchen. Fettdegeneration der Leberzellen ist stellenweise ganz charakteristisch, vacuoläre in geringerem Grade. In den Gallengängen sind entzündliche Prozesse wahrscheinlich, man erblickt stellenweise im Epithel derselben zahlreiche Leukocyten, auch tritt deutlich eine Desquamation des Epithels zu Tage. Die Gallengänge erscheinen größtenteils leer, nur wenige enthalten hyaline Massen mit Kernen darin. Gallengangs- oder Bindegewebswucherung ist nirgends zu erkennen. 
Bei Abfassung dieser Arbeit, welche einer Anregung des Herrn Prof. L. Brauer ihre Entstehung verdankt, hatte sich Verfasser der freundlichen Unterstützung nicht nur dieses Herrn, sondern auch des Herrn Prof. Otto Cohnheim zu erfreuen. Ihnen, sowie Herrn Prof. Kossel, dessen freundlichem Entgegenkommen Verfasser die Erlaubnis zur Anstellung seiner Versuche im hiesigen physiologischen Institut verdankt, gestattet er sich hiermit, schuldigen Dank auszusprechen.

\title{
Bemerkung zu der Erwiderung von Martin Krüger.
}

\author{
Von \\ Otto Folin.
}

(Der Redaktion zugegangen am 14. Jan. 1904.)

Gegen die Erwiderung von Martin Krüger Bd. XL, S. 316 dieser Zeitschrift möchte ich nur anführen, daß es sich in der erwähnten Frage nicht um «Prioritätsrechte \ handelt. Es ist uns ganz klar, daß die Krü gerReichschen Versuche unabhängig von den in meinem Laboratorium ausgeführten Versuchen Shaffers waren. Daß auch wir bis zur Veröffentlichung dieser Versuche ohne Kenntnis des in einer Breslauer Inaugural-Dissertation beschriebenen Verfahrens waren, hat ja Krïgor auch nicht bestritten. Es ist aber klar, daß Krüger und Reich, ebenso wie Schittenhelm, zur Zeit als sie ihre Arbeiten zum erstenmale in einer wissenschaftlichen Zeitschrift publizierten, von den genannten Versuchen Shaffers Kenntnis haben mußten. Daß sie unter solchen Umständen der Abhandlung Shaffers, die mindestens ebenso zweckmäßige Versuche und eine weit eingehendere Kritik der in Frage stehenden Methodik zur Ammoniakbestimmung enthält, mit keiner Silbe Erwähnung tun, bleibt mir auch jetzt unverständlich. 